\title{
Solid-State Conductive-Bridging Reconfigurable RF-Encoding Particle for Chipless RFID Applications
}

\author{
M.P. Jayakrishnan, Arnaud Vena, Brice Sorli and Etienne Perret, Senior Member, IEEE
}

\begin{abstract}
We report the design and first results of a ReConfigurable RF-encoding particle (REP) for Chipless RFID tag, based on a Nafion-CBRAM Cell. The developed tag consists of a modified shorted dipole as REP, equipped with a CopperNafion ${ }^{\circledR}$-Aluminum Metal-Insulator-Metal (MIM) non-volatile Ionic-Bridging switch. The MIM switch is similar to an RC parallel network, which tunes the electrical length of the resonator. The Chipless tags switch between two different resonance frequencies, in the Set and Reset state of the switch. An electrical model is also developed for the REP, to explain the mechanism of switching. The experimental results are in close agreement with the simulated results using commercial EM Simulators. This study is a proof of concept of electronically reconfigurable Chipless tags based on integrated switches which have the potential to be directly printed along with the tag. Keywords - CBRAM, MIM Switch, RF Switch, Chipless RFID
\end{abstract}

\section{INTRODUCTION}

$\mathrm{R}$ FID is one of the most influential inventions of the 20th century. This technology is now mature enough to be used for a variety of applications like consumer products in supermarkets, health monitoring applications in the medical field, item level tracking for Internet-of -things application etc. [1] - [4]. Yet a hindering factor to the widespread use of this technology is the cost per unit tag. Even though Chipless RFID technology has brought in revolutionary changes in this aspect, this technology is still dominated in application by the optical barcode technology due to its flexibility in use and lower cost. Nevertheless, it is certain that current dedication of researchers all over the world on Chipless RFID, and the advances in disposable printed electronics would uplift it to the technology mainstream in a short period of time, backed by ingenious functionalities like large read range without optical contact, sensor integration functionality,

Manuscript received Nov. 13, 2017; revised Feb. 14, 2018 and accepted Mar. 28, 2018. This work is supported in part by the Grenoble Institute of Technology, Université Grenoble Alpes and the Institut Universitaire de France.

M.P. Jayakrishnan and Etienne Perret is with the Laboratoire de Conception et d'Intégration des Systèmes, Grenoble INP, Université Grenoble Alpes, 26902 Valence, France. Email: jayakrishnan.mp@1cis.grenoble-inp.fr, etienne.perret@1cis.grenoble-inp.fr. Etienne Perret is also a member of Institut Universitaire de France, 75005 Paris, France.

Arnaud Vena, and Brice Sorli are with the Institut d'Electronique et Systèmes, Université de Montpellier 2, Montpellier 34095, France. Email: arnaud.vena@umontpellier.fr, brice.sorli@ies.univ-montp2.fr reconfigurability etc. which cannot be implemented with the current technology of optical barcodes. The reconfigurability of Chipless RFID is a key feature which distinguishes this technology from the optical barcodes and opens the way for new applications. The possibility to reconfigure helps in reducing the cost per unit, by bulk manufacture, and appreciates tag reuse, as the encoding could be done at the time of application by the consumer. Fig. 1 shows the concept of using a single resonator (shorted dipole) with a switch to represent two different spectral signature combinations, which means two different tag's ID. This concept is called Frequency Shift (FS) coding [3] and the coding capacity $N$ per tag is given by (1). Where $\Delta f$ is the bandwidth per resonator, $d f$ is the frequency step per switch position per resonator, and $k$ is the number of resonators on the tag. One should also note that, here, as only one switch is implemented per resonator in Fig. 1 , two different frequencies of resonance are obtained, so the $\Delta f$ could be chosen to be equal $2 d f$.

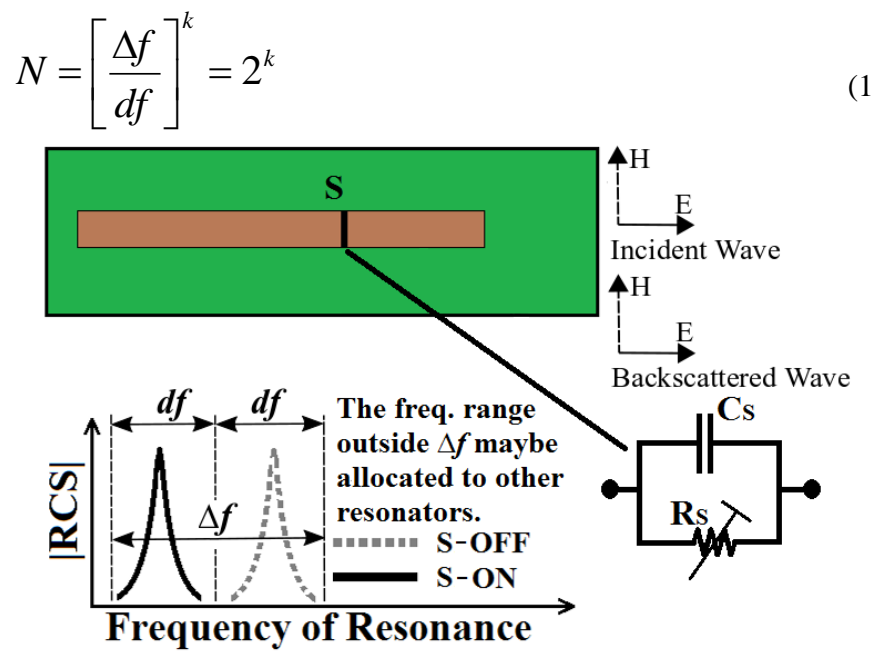

Fig. 1 The concept of encoding data in reconfigurable RFID tags using FS coding technique for one $(k=1)$ resonator, where $R_{s}$ can switch between two values: low and high resistance so $N=2$.

The idea of reconfiguring a resonator as shown in Fig. 1 can be implemented in several configurations using multiple switches on resonators to form multi-bit Chipless tags. Jalali et al. [5] has reported RFID tags based on capacitively tuned split-dipoles, in which if the capacitance is replaced with a switch as explained here, could evolve to a reconfigurable RFtag. Similarly, a switch could also be used to reconfigure the electrical length of a resonator, as proposed in this study. 
A few articles have reported the concept of modifying the information encoded in the Chipless RFID tag [5] - [7]. This is done by modifying the dimensions of the resonating elements by external methods like conductive inks and lumped elements and are irreversible, making the tags one time programmable. However, a convenient and reliable technology for electronically reconfigurable Chipless RFID tags (write and rewrite feature) is not yet claimed by any authors.

In this article, for the first time, we present the concept and first results of the realization of an electronically reconfigurable solid state REP for Chipless RFID. The presented device is based on a non-volatile memory switching technology called Conductive Bridging Random Access Memory (CBRAM) or Metal-Insulator-Metal (MIM) switch [8] - [11]. Here we use an MIM switch with Copper (active) and Aluminum (passive) electrodes, and Nafion ${ }^{\circledR}$ as electrolyte [11]. This approach would certainly be an excellent breakthrough for Chipless RFID technology [2], as the used switch is directly integrated into the tag and has the potential to be printed along with the tag. This method would be superior in performance in comparison to the integration of classical lumped element switches into tags via soldering, which would tend to degrade the efficiency to some extent.

\section{DESIGN AND MECHANISM OF OPERATION}

The REP design for RFID tag is inspired from the wellknown 'hairpin' or ' $\mathrm{C}$ ' resonator [4]. The REP resonates similar to a half wavelength shorted dipole, when excited by an electric field polarized along the $\mathrm{Y}$ axis as shown in the Fig. 2. Two tags are designed with resonators each of different electrical lengths for validation of the mechanism.

The design is first simulated using the CST Microwave Studio. A lumped resistor similar to the measured filament resistance values of the MIM switch $(0.5 \Omega$ and $30 \mathrm{M} \Omega)$ is used to change the state of the tag. Response of simulation of both tags, without the switch, is similar to the Set state response.

Topology of the resonators and photograph of the fabricated tags are given respectively in Fig. 2 and 3. The tags are fabricated on a common FR4 substrate using simple fabrication steps in ambient environment without the use of any clean room technologies. The fabrication process has been optimized for the realization of an RF switch [11]. The reported process is compatible even for flexible substrates like paper (real world substrates for chipless RFID tags), with some slight modifications. Where, the electrodes would be formed totally by thermal vapor deposition or inkjet printing, and no process temperature limitation is observed with the technique. The MIM switch is integrated to the resonator as shown in Fig. 2, by tapering the width to reduce effective capacitance of overlapping sections. The switch is formed with Copper as active electrode and Aluminum as inert electrode, both sandwiching a $600 \mathrm{~nm}$ layer of Nafion as the electrolyte/Ion conductor. On application of a positive voltage with respect to the copper electrode, copper ions migrate through the electrolyte to form a conductive filament to the passive electrode (Aluminum) establishing the set/closed state [11]. Similarly, a voltage pulse of reverse polarity would reverse the process and breaks this filament, leaving the switch in reset/open state. DC voltage pulses used to operate the switch are generated from a Keithley 2450 source meter. The switch is set using a DC ramp of $12 \mathrm{~V} / 0.5 \mathrm{~mA}$ and Reset instantaneously at $-20 \mathrm{~V} / 0.15 \mathrm{~A}$. The voltage pulses used for the set/reset of the MIM switch are given in Fig. 4.
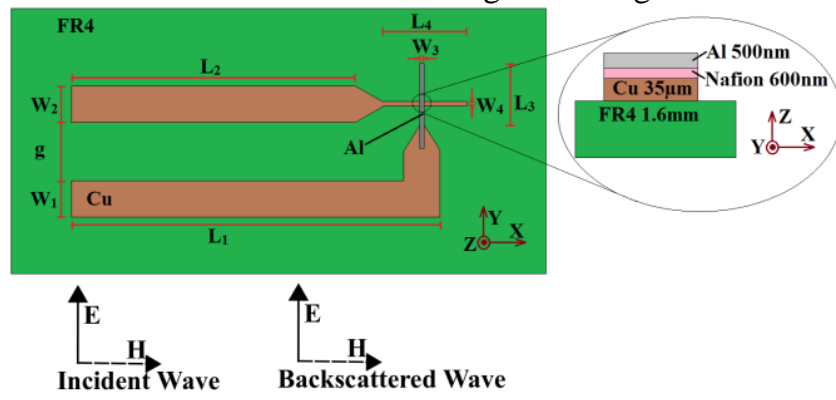

Fig. 2. Topology of the RF-Tag. Tag 1: $W_{1}=W_{2}=3 m m, W_{3}=0.4 \mathrm{~mm}$, $\mathrm{W}_{4}=0.16 \mathrm{~mm}, \mathrm{~g}=3 \mathrm{~mm}, \mathrm{~L}_{1}=21.5 \mathrm{~mm}, \mathrm{~L}_{2}=14.5 \mathrm{~mm}, \mathrm{~L}_{3}=1.8 \mathrm{~mm}, \mathrm{~L}_{4}=4 \mathrm{~mm}$. Tag 2: $\mathrm{W}_{1}=\mathrm{W}_{2}=3 \mathrm{~mm}, \mathrm{~W}_{3}=0.4 \mathrm{~mm}, \mathrm{~W}_{4}=0.16 \mathrm{~mm}, \mathrm{~g}=3 \mathrm{~mm}, \mathrm{~L}_{1}=37 \mathrm{~mm}, \mathrm{~L}_{2}=30 \mathrm{~mm}$, $\mathrm{L}_{3}=1.8 \mathrm{~mm}, \mathrm{~L}_{4}=4 \mathrm{~mm}$.

In order to ensure a smooth set of the switch a positive growing current limited voltage ramp waveform is used. The switch is set in the presented case at $10 \mathrm{~V}$, as shown by the abrupt fall in measured voltage in Fig. 4. A lumped model of the tag derived from [3] is optimized using the PSpice software and is given in Fig. 5.

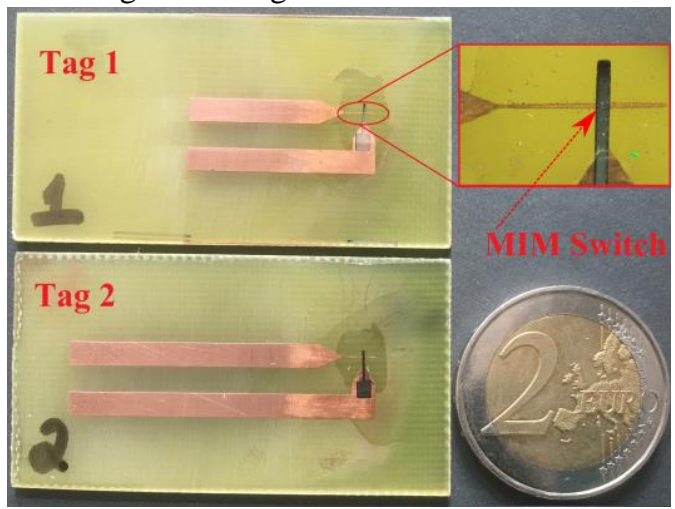

Fig. 3. Photograph of the Fabricated Tags, with microphotograph in the inset showing the MIM switch area.

The MIM switch could be modeled as an RC parallel network [11]. The equivalent electrical model of the ' $\mathrm{C}$ ' resonator without the switch could be defined as a combination of RLC serial networks each for the antenna mode and the structural mode [3]. In this case, electrical equivalent of the MIM switch is inserted to RLC equivalent for the antenna mode, as it is responsible for the shift in resonance frequency. $\mathrm{C}_{\mathrm{MIM}}$ is the equivalent parallel plate capacitance of the MIM switch (calculated parallel plate capacitance due to the electrode surface, electrolyte thickness and dielectric constant) and $\mathrm{R}_{\mathrm{MIM}}$ is the measured DC resistance of the MIM switch, other lumped elements are optimized through fitting, with experimental results. The values of the electrical model for both tags are given in Table1.Mechanism of operation of the tags could be explained as follows. When the switch is set, filament resistance $\left(\mathrm{R}_{\mathrm{MIM}}\right)$ falls to a very low value $(0.5 \Omega)$, which in effect short circuits the $\mathrm{C}_{\mathrm{MIM}}$, exhibiting set state resonance. Inversely when the switch is reset, the filament link is broken and $\mathrm{R}_{\mathrm{MIM}}$ is a large value $(30 \mathrm{M} \Omega$ ) and can be neglected from the circuit, thus $\mathrm{C}_{\text {MIM }}$ adds to the current path 
lowering the effective capacitance, thereby shifting the resonance to a higher frequency.

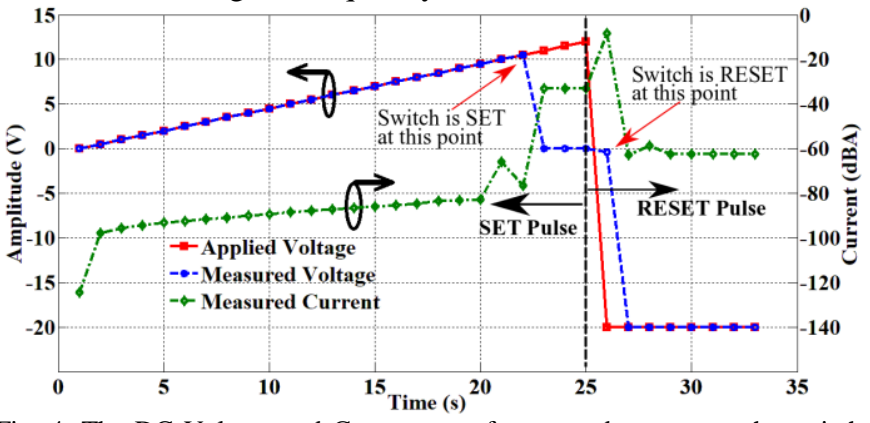

Fig. 4. The DC Voltage and Current waveforms used to operate the switch. The switch is Set at $22 \mathrm{~s}$ and is maintained between $22 \mathrm{~s}$ and $26 \mathrm{~s}$ and Reset after 26s.

TABLE I

ELECTRICAL MODEL OF THE RECONFIGURABLE TAG

\begin{tabular}{cccccc}
\hline \hline Parameters & Tag1 & Tag 2 & Parameters & Tag1 & Tag2 \\
\hline $\mathrm{R}_{\mathrm{S}}$ & $20 \Omega$ & $20 \Omega$ & $\mathrm{R}_{\mathrm{A}}$ & $5 \Omega$ & $8 \Omega$ \\
$\mathrm{L}_{\mathrm{S}}$ & $1 \mathrm{nH}$ & $1 \mathrm{nH}$ & $\mathrm{L}_{\mathrm{A}}$ & $14 \mathrm{nH}$ & $35 \mathrm{nH}$ \\
$\mathrm{C}_{\mathrm{S}}$ & $0.2 \mathrm{pF}$ & $0.2 \mathrm{pF}$ & $\mathrm{C}_{\mathrm{A}}$ & $0.43 \mathrm{pF}$ & $0.473 \mathrm{pF}$ \\
$\mathrm{V}_{\mathrm{S}}$ & $30 \mathrm{mV} / 0^{\circ}$ & $30 \mathrm{mV} / 0^{\circ}$ & $\mathrm{R}_{\mathrm{MIM}}$ & $0.5 \Omega$ & $0.5 \Omega$ \\
$\mathrm{V}_{\mathrm{A}}$ & $1.5 \mathrm{mV} / 0^{\circ}$ & $1.5 \mathrm{mV} / 0^{\circ}$ & $\mathrm{C}_{\text {MIM }}$ & $1.98 \mathrm{pF}$ & $1.98 \mathrm{pF}$ \\
\hline
\end{tabular}

$\mathrm{R}_{\text {Ant }}$ is a small value resistance for connecting the Current/Voltage probe in PSpice.

\section{RESULTS}

RF responses of the tags were measured using Agilent N5222A Network Analyzer in a bi-static radar setup [4] using two wideband horn antennas in Co-Polarization. The measured RCS backscatter of the tags along with results of simulation, using the electrical model and full wave simulation are given in Fig. 6. The obtained RCS magnitude is high enough to be recognized with a tag reader in real environment with read range of around $30 \mathrm{~cm}$ [4].Both tags 1 and 2 shows a separation of $200 \mathrm{MHz}$ and $140 \mathrm{MHz}$ respectively, between set and reset state resonance. The experimental results are in agreement with results of the lumped model and full wave simulation.

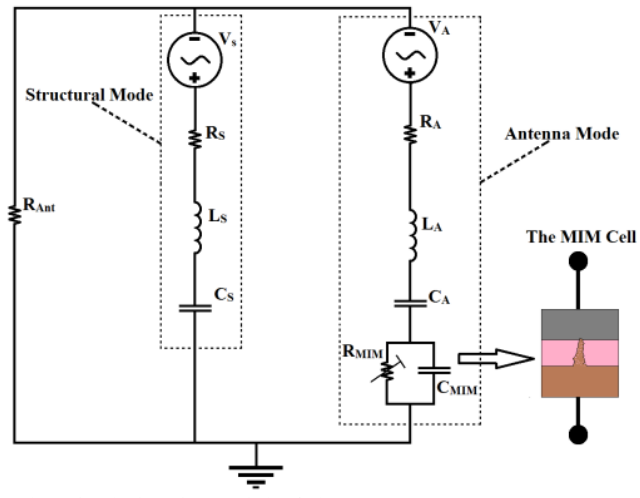

Fig. 5. The electrical circuit model of the REP.

\section{CONCLUSION}

We have successfully demonstrated the potential ability of CBRAM/MIM switch for solid state reconfigurable tags. Lumped model of the presented tag gives a good physical insight to the operating mechanism of the resonators. The measured best Set/Reset resistances of the switch are $0.5 \Omega$ and $30 \mathrm{M} \Omega$ respectively. The presented resonator represents only one bit, and five such resonators could be integrated to a credit card sized tag. However, more number of MIM switches could be added to a single resonator to increase the number of bits per tag. Both of the tags experimented were switched for more than 50 cycles and are still functional. This is a good number of repetitions for tags in applications like transport tickets, and this is the first time that a chipless RFID tag with non-volatile electronic reconfigurability of its ID is realized, to the best of our knowledge.

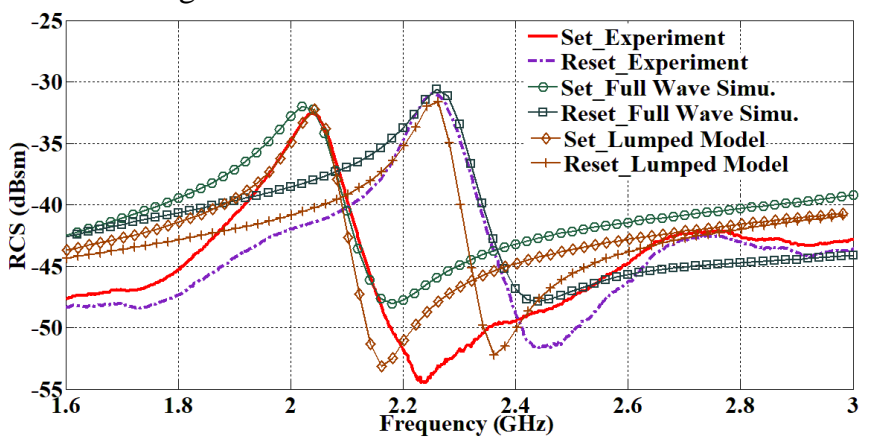

(a)

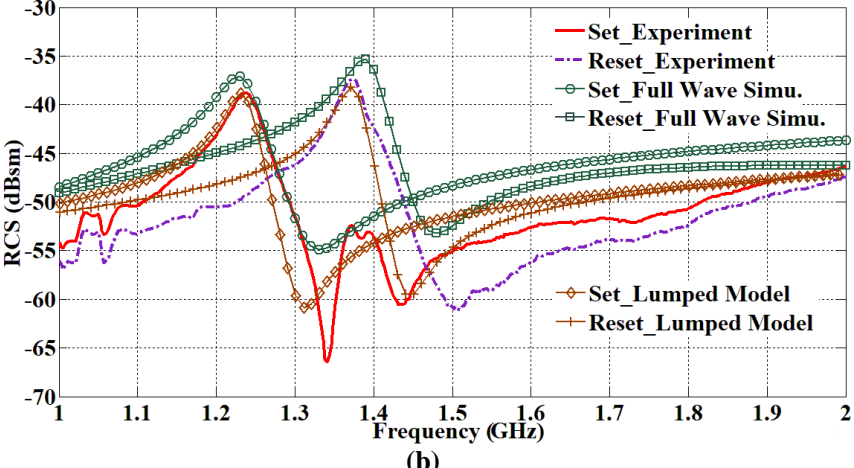

(b)

Fig. 6 |RCS| response of Tag1 (a), and Tag2 (b).

\section{REFERENCES}

[1] S. Tedjini et al., "Hold the Chips: Chipless Technology, an Alternative Technique for RFID”, IEEE Microw. Mag., vol. 14, no. 5, pp. 56-65, 2013.

[2] E. Perret, "Radio Frequency Identification and Sensors: From RFID to Chipless RFID”, Wiley-ISTE, 2014. ISBN: 978-1-84821-766-9

[3] A. Vena et al., "Chipless RFID based on RF Encoding Particle", ISTEElsevier, 2016. ISBN: 978-1-78548-107-9.

[4] A. Vena et al., "Chipless RFID Tag Using Hybrid Coding Technique," in IEEE Trans. Microw. Theory Techn., vol. 59, no. 12, pp. 3356-3364, 2011.

[5] I. Jalaly et al., "Capacitively-tuned split microstrip resonators for RFID barcodes," 2005 European Microwave Conference, 2005

[6] A. Vena et al., "A Novel Near-Transparent ASK-Reconfigurable InkjetPrinted Chipless RFID Tag," in IEEE Antennas Wireless Propag. Lett. , vol. 12, pp. 753-756, 2013.

[7] L. Zheng et al., "Design and implementation of a fully reconfigurable Chipless RFID tag using Inkjet printing technology," 2008 IEEE Int. Symp. Circuit Syst., Seattle, WA, pp. 1524-1527.

[8] J. Nessel et al., "Chalcogenide Nanoionic-Based Radio Frequency Switch", U.S. Patent, US 7923715 B2, Apr. 12, 2011.

[9] A. Vena et al., "A fully passive RF switch based on nanometric conductive bridge," 2012 IEEE/MTT-S Int. Microw. Symposium Dig., Montreal, QC, Canada, pp. 1-3, 2012.

[10] E. Perret et al.," Realization of a Conductive Bridging RF Switch Integrated onto Printed Circuit Board", Progress in Electromagn. Res., vol. 151, pp. 9-16, 2015.

[11] M.P. Jayakrishnan et al., "Nafion Based Fully Passive Solid State Conductive Bridging RF Switch", IEEE Microw. Compon. Lett. , vol. 27, no. 12, pp. 1104-1106, 2017. 